\title{
Modeling of reaction kinetics in generation of hydrogen from wastewater by microbial electrolysis
}

\author{
Ujwal Shreenag Meda ${ }^{1}$, Lourdu Antony Raj Molayan Amritanatan ${ }^{1}$, and Kruthika Parappa ${ }^{2}$ \\ ${ }^{1}$ Department of Chemical Engineering, RV College of Engineering, Bengaluru, India \\ ${ }^{2}$ Department of Biotechnology, RV College of Engineering, Bengaluru, India
}

\begin{abstract}
High carbon footprints and limited availability of fossil fuels have motivated researchers to find alternatives to fossil fuels and the ways of producing them. Hydrogen is an alternative fuel and can be generated by electrohydrogenesis in a Microbial Electrolysis Cell (MEC) using wastewater. At times, the microorganisms known as exoelectrogens are added externally to the wastewater in the form of biomass. Biomass serves as a parameter to optimize the yield of hydrogen. In this research work an attempt is made to understand the effect of the biomass concentration on the substrate utilization by the exoelectrogens and product formation. This research work also aims at studying the biochemical reaction kinetics and to identify a model that best describes the kinetics of the reactions involved, at the electrodes. It was observed that on increasing the biomass concentration from $0.7 \mathrm{~g} / \mathrm{L}$ to $1.4 \mathrm{~g} / \mathrm{L}$, the gas liberation rate increased from $9.42 \mathrm{ml} /$ day to $15.33 \mathrm{ml} /$ day and substrate utilization increased from $86.8 \%$ to $94.3 \%$. This was in close agreement with the solution of the identified model. It was found out that the energy efficiency of MEC improved substantially by $30 \%$ and the energy demand was decreased by $38.5 \%$ when the initial biomass concentration was doubled.
\end{abstract}

\section{Introduction}

Energy is an indispensable yet exhaustible commodity in the modern world. Conventional fuels, which are nonsustainable resources, are used to fulfill the majority of energy needs, which at some point may exhaust completely. The ever-increasing demand for energy has prompted researchers to find renewable and sustainable alternatives for meeting the growing energy demand and the serious quandary of energy scarcity. Many tireless endeavors are being made to harness renewable energy from sources such as solar, geothermal, and wind energy. There is immense potential in technologies which make use of wastewater to generate electricity or fuel. These technologies can produce power on-site for water treatment facilities in even the most isolated places around the world. They take developing nations a step forward towards providing clean water [1]. One such technology is the use of Microbial Electrochemical Systems. Electrochemical systems can be used as alternatives to recover energy from organic waste by catalyzing electrochemical reactions using microbes [2].

MECs, are new and emerging technology related to Microbial Fuel cells, wherein, microorganisms are used to generate hydrogen. The organic matter present in the wastewater is broken down to produce hydrogen on an application of external voltage [3, 4]. A special class of microbes called exoelectrogens is used in MECs which produce and conduct electrons by the formation of a nanowire in a biofilm [5]. The setup consists of two electrodes; anode and a cathode. The electrodes are separated from each other and the anode chamber is where the microbial reaction takes place since it contains the microbes and the substrate [4]. The proliferation of microbes leads to the formation of a biofilm. MECs require supplementary input of voltage added externally to enable hydrogen formation at cathode during the metabolic process [6]. As the reaction proceeds, the concentration of the biomass increases while the substrate concentration decreases with time. The reactions shown in equation 1 and 2 , take place at the electrodes of the MEC respectively under standard conditions. For electro-hydrogenesis, acetate is used as a model electron donor $[4,7]$.

Anode reaction:

$$
4 \mathrm{H}_{2} \mathrm{O}+\mathrm{CH}_{2} \mathrm{COO} \leftrightarrow 9 \mathrm{H}^{+}+2 \mathrm{HCO}+8 e^{-}
$$

Cathode reaction:

$$
2 H^{+}+2 e^{-} \leftrightarrow H_{2}
$$

Since microbes are involved, the reactions are biochemical in nature. Attempts are made to understand the mechanism of hydrogen evolution reaction in a

* Corresponding author: ujwalshreenagm $@$ rvce.edu.in 
single chambered MEC [8]. The effect of enzymatic degradation on halogenated organic pollutants in a synthetic wastewater was explored [9]. Theoretical aspects of thermodynamics and kinetics are discussed and the practical aspects that are poorly investigated are listed [10]. Investigation on current, concentration and potential distribution inside MEC and their impact on distribution of overvoltages is carried out [11]. Mathematical models describing the response of current in an MEC are proposed [12]. Studies on kinetic parameters of a specific microbial community are made in a MFC [13]. Little information is available in the literature on the kinetics of the reactions that occur in MEC and conventional kinetic models cannot be used to study the reaction kinetics. The primary deviation is the inclusion of growth kinetics because of the presence of living organisms, for the reasons that (i) size and shape of the cells can vary largely (ii) the total concentration might remain constant while there is a change in the number of cells or vice-versa (iii) the growth culture is a heterogeneous mixture of young and old cells, which is continuously changing. Models that account for both growth kinetics and reaction kinetics should be considered and the kinetic parameters can be determined experimentally or by simulation [14].

Considering biomass concentration as a parameter that influences the performance of MEC, it was observed that product concentration and the product formation rate was directly proportional to the amount of biomass.

\section{Materials and methods}

The experiment was conducted in two runs to study the effects of varying initial biomass concentration on substrate utilization and product formation. The initial biomass concentrations were kept to be $0.7 \mathrm{~g} / \mathrm{L}$ and $1.4 \mathrm{~g} / \mathrm{L}$ by keeping other parameters like the substrate concentration, temperature and $\mathrm{pH}$ constant.

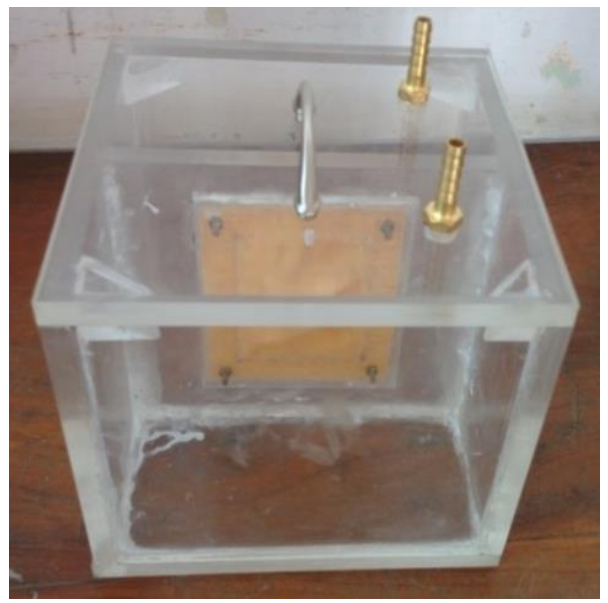

Fig. 1:Dual chamber Microbial Electrolysis Cell fabricated using acrylic material and CMI - 7000 membrane

\subsection{Fabrication of MEC}

MEC was constructed with acrylic sheets. A ratio of surface area of graphite anode to stainless steel cathode was maintained at 1:2 with a spacing of $6 \mathrm{~cm}$ in between. The two chambers were separated using CMI - 7000 membrane [15] as shown in Fig. 1. A potential difference of $0.9 \mathrm{~V}$ was maintained using a regulated power supply. The electrolytes used in the anode and cathode were the anolyte and distilled water respectively. Anolyte consists of carbon -rich substrate and microorganisms capable of forming a biofilm.

\subsection{Preparation of anolyte}

The electrolyte of the anode chamber consists of the microbial culture, buffer and the substrate. Each of the components of the anolyte was prepared separately as follows.

\subsubsection{Preparation of microbial Culture}

The bacteria Pseudomonas Aeruginosa was used as a microbe to breakdown the substrate in the anode chamber. The growth medium for the microbes was prepared by dissolving $2.6 \mathrm{~g}$ of broth powder in $200 \mathrm{ml}$ of distilled water using a conical flask. The broth powder consisted of $5 \mathrm{~g} / \mathrm{L}$ of $\mathrm{NaCl}$ and an equal quantity of peptic digest of animal tissue. Also, it contained $1.5 \mathrm{~g} / \mathrm{L}$ of yeast and an equal quantity of Beef Extract. Later it was autoclaved for $60 \mathrm{~min}$ at a pressure of $15 \mathrm{psi}$.

The Pseudomonas aeruginosa culture was inoculated $(0.7 \mathrm{~g} / \mathrm{L}$ and $1.4 \mathrm{~g} / \mathrm{L})$ i.e., transferred to the media inside a Laminar Air Flow (LAF) to maintain an aseptic environment [16]. Later it was incubated by subjecting it to continuous mixing using an orbital shaker for 3 days. The turbidity of the solution increased indicating the growth of the bacteria. Later the culture was kept in a refrigerator at $10^{\circ} \mathrm{C}$ for later use in the anolyte.

\subsubsection{Preparation of substrate}

Sodium acetate was used as the substrate for the reason that it is the most common product obtained after fermentation. $10 \mathrm{~g}$ of sodium acetate was added to 250 $\mathrm{ml}$ distilled water to produce the substrate, of concentration $0.29 \mathrm{M}$ sodium acetate solution equivalent to $40 \mathrm{~g} / \mathrm{L}$.

$1000 \mathrm{ml}$ of anolyte was prepared by mixing $250 \mathrm{ml}$ of $0.29 \mathrm{M}$ sodium acetate solution and $60 \mathrm{ml}$ of the microbial culture. The volume was made up to $1000 \mathrm{ml}$ using tap water. $\mathrm{pH}$ of anolyte was found to be 8.0 using a $\mathrm{pH}$ meter. The $\mathrm{pH}$ was made to 7.0 by the addition of a phosphate buffer solution which was previously prepared [17]. To maintain an anaerobic condition in the anode chamber, nitrogen gas was continuously sparged into the chamber throughout the experiment.

\subsection{Determination of Biomass concentration $(X)$ in the anolyte}


Measurement of biomass concentration was primarily based on estimating the turbidity using UV-VIS spectrophotometry by the principle of Beer-Lambert's law. The absorbance of aliquots with varying biomass concentration was determined and a calibration chart was prepared by plotting the absorbance versus biomass concentration.

Once the hydrogen generation experiment in MEC started, $10 \mathrm{ml}$ of the anolyte (contents of anode chamber of MEC) was drawn at regular time intervals (1 day) as shown in Fig. 2. The absorbance was measured at 601 $\mathrm{nm}$ and the corresponding concentration of biomass was determined using the calibration chart. Later the $10 \mathrm{ml}$ solution was subjected to centrifugation at $5000 \mathrm{rpm}$ for 15 minutes in a plastic test tube. Biomass was obtained as a pellet and the rest remained as a supernatant liquid. The supernatant liquid was separated and used to determine the concentration of the substrate. Finally, the centrifuged pellet was resuspended in $10 \mathrm{ml}$ of distilled water, $0.1 \mathrm{~g}$ of sodium acetate was added, mixed well and the mixture was reinjected to the anode chamber of MEC.

Biomass concentration was calculated as shown in equation 3.

$$
X=\frac{\text { Weight of dried pallet, } W}{\text { Volume of solution drawn }(100 \mathrm{ml})}
$$

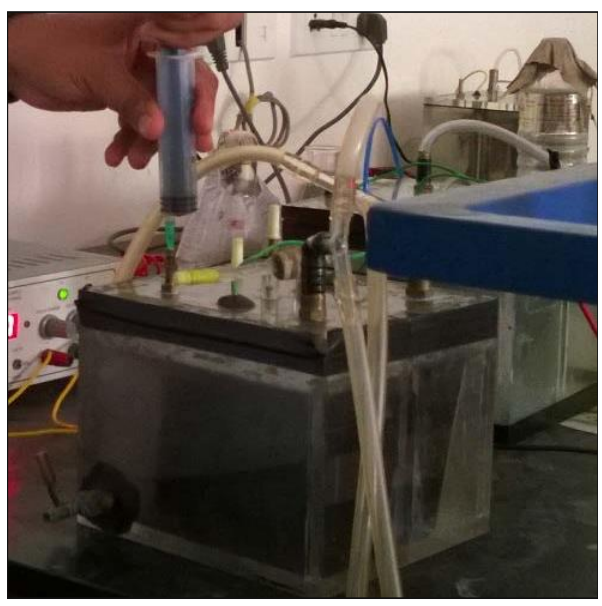

Fig. 2: Sample collection for analysis, from the anode chamber of MEC during the experiment using a syringe

\subsection{Determination of substrate concentration (S)}

The substrate was analyzed using the principle of volumetric analysis. It is carried out by titrating the supernatant liquid against $0.104 \mathrm{M}$ Hydrochloric acid in the presence of methyl orange indicator and the $\mathrm{pH}$ was measured using a $\mathrm{pH}$ meter. The concentration of sodium acetate in the supernatant was determined using the recorded titer value.

\subsection{Determination of Product concentration}

Although the exact composition of the individual gases in the gas mixture evolved at cathode can only be estimated by a Gas Chromatograph, the overall concentration of the gases evolved can be determined by using fluid statics and the Ideal Gas Equation.

\subsubsection{Gas collection mechanism}

Gases evolved at anode and cathode were collected in 2 separate glass burettes, by the principle of downward displacement of water. This system is often referred to as a respirometer. Plastic pipes were used to connect MEC chambers with the burettes.

\subsubsection{Procedure for determination of gas composition}

The pressure of the residual gases inside the burette $P_{0}$ was calculated when the length of the water column in burette was $100 \mathrm{~cm}$ as shown in Fig. 3. Once the experiment was begun, the length of water column $h$ in burette was noted on a daily basis and new value of pressure was calculated as $P$ (actual pressure). Difference between $P_{0}$ (pressure at water level of $0 \mathrm{~cm}$ ) and $P$ was calculated as $\Delta P . \Delta P$ was substituted in the Ideal Gas Law equation and concentration of the gas was calculated where $P_{a}$ is the atmospheric pressure.

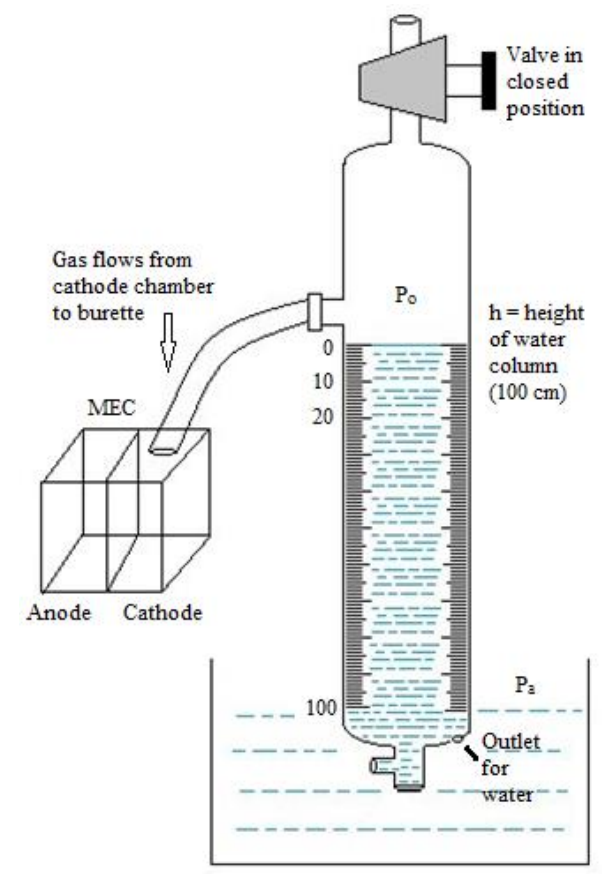

Fig. 3: Schematic representation of gas collection mechanism with water level at $0 \mathrm{~cm}$ mark and $h=100 \mathrm{~cm}$

Applying the principle of hydrostatic equilibrium at 100 cm mark,

$$
\begin{aligned}
& P_{0}+\rho h g_{0}=P_{a} \\
& P_{0}=P_{a}-\rho h g_{0}
\end{aligned}
$$

Again, applying Principle of hydrostatic equilibrium at $85 \mathrm{~cm}$ mark, 


$$
\begin{gathered}
P+\rho g h_{85}=P_{a} \\
P=P_{a}-\rho g h_{85} \\
\Delta P=P-P_{0}
\end{gathered}
$$

The concentration of gas,

$$
C=\frac{\Delta P \cdot M}{R T} g / L
$$

where $M, R$ and $T$ are average molecular weight of gaseous mixture in $\mathrm{g} / \mathrm{mol}$, universal gas constant in L.atm/mol.K and temperature in $K$ respectively.

At the end of the experiment, the gaseous sample was analyzed in a gas chromatograph and the percentage of hydrogen was found to be around $51 \%$ and the rest being nitrogen $(29 \%)$, carbon dioxide $(15 \%)$ and oxygen $(5 \%)$. The same composition was utilized for calculating the average molecular weight of the gaseous mixture.

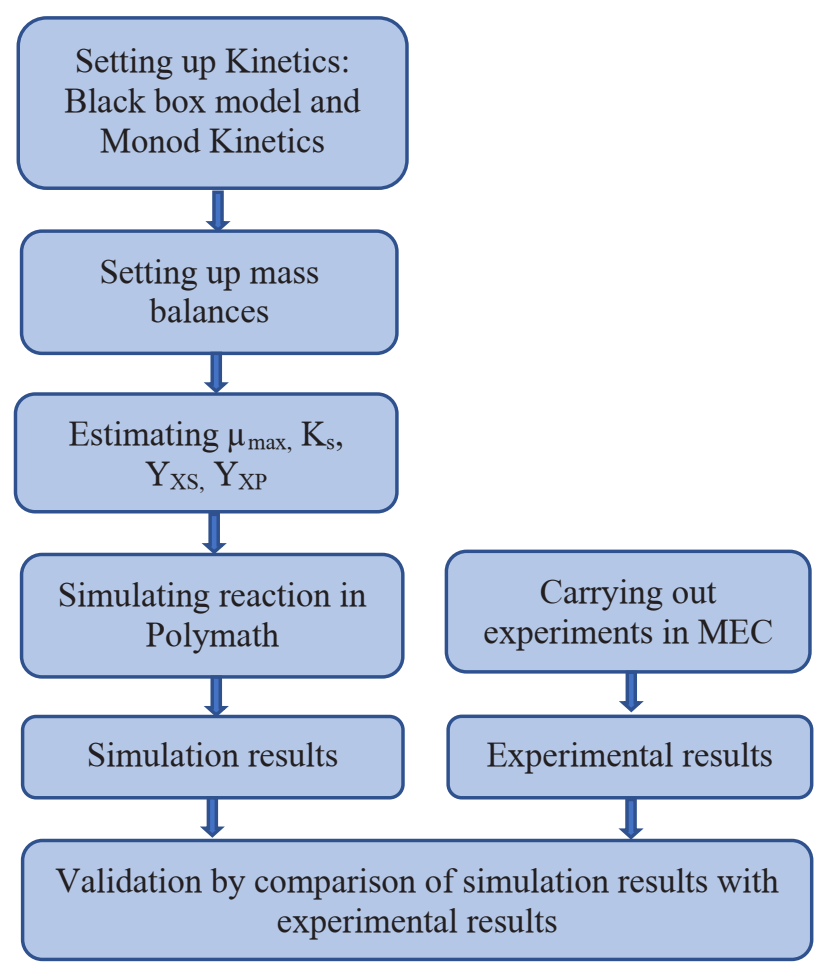

Fig. 4: Steps involved in building and validation of kinetics model

\section{Modeling of Reaction Kinetics}

An attempt was made to model the kinetics of reactions taking place in the anode chamber of MEC. The general workflow is shown in Fig. 4. Biochemical reaction kinetics models vary from conventional reaction kinetics models. Various models are available which closely represent the growth kinetics. One such relevant kinetic model is unstructured growth kinetics model.

In these unstructured models, all cellular components are clubbed into one biomass component represented as Total Concentration of Biomass, X. A single reaction describes the overall conversion of substrate into biomass. The kinetics of this single reaction is represented by the specific growth rate of biomass, $\mu$. A black box model is an unstructured model which is a mathematical representation of biomass growth. According to the black box model yield of biomass with reference to substrate and yield of all other compounds consumed and produced by the cells are constant [18]. The specific substrate consumption rate, $-r_{s}$, is proportional to $\mu$ according to the equation 10 .

$$
-r_{s}=Y_{X S} \cdot \mu
$$

It is observed that cell growth, on a single limiting substrate, in terms of $\mu$, is proportional to substrate concentration, $S$, at low concentrations. At higher concentrations of $S, \mu$ attains maximum value and remains constant and this can be described by Monod model $[14,19]$ represented by equation 11 .

$$
\mu=\mu_{\max } \frac{S}{S+K_{s}}
$$

Where $K_{s}$ is Monod half saturation constant, $\mathrm{g} / \mathrm{L}$ and $\mu_{\max }$ is the maximum specific growth rate of microbes per day determined using Lineweaver Burk plot from the experiments carried out [2]. Following are the assumptions made.

1. The reactions occurring in the anode chamber of MEC are well described by the Black Box model.

2. There is only one limiting substrate, Sodium Acetate, the only carbon source which is consumed by the microbes for their growth.

3. The disappearance of all other substrates and generation of metabolic products \& biomass is found by mass balances using yield coefficients.

4. The substrate is uniformly distributed in the anodic compartment (ideal mixing) and substrate/acetate gradient on biofilm is neglected.

5. $\mathrm{pH}$ and temperature of the solution at the anode are constant.

6. Instant transfer of constituents from the liquid to gaseous phase is assumed.

Following are the model equations,

For $S$,

$$
-Y_{X S} \cdot \mu \cdot X=\frac{d s}{d t}
$$

For $X$,

$$
\mu \cdot X=\frac{d x}{d t}
$$

For $P$,

$$
Y_{X P} \bullet \mu \cdot X=\frac{d P}{d t}
$$

where, 


$$
\begin{gathered}
Y_{X S}=\frac{\Delta S}{\Delta X} \\
Y_{X P}=\frac{\Delta P}{\Delta X}
\end{gathered}
$$

$\mu_{\max }, K_{s}, \quad Y_{X S}$ and $Y_{X P}$ vary with initial biomass concentration. Polymath software version 5.1 was used to solve the model using RK45 (Runge Kutta) method and to simulate the variation of substrate, biomass and product concentrations with time.

\section{Results and Discussion}

Two experiments were conducted in double chamber MEC by varying initial biomass concentration and keeping all other parameters constant such as type of substrate (sodium acetate), initial substrate concentration, $S_{0}=9.92 \mathrm{~g} / \mathrm{L}$, electrode type (graphite anode and stainless-steel cathode), electrode spacing (6 $\mathrm{cm})$ and electrode potential $(0.9 \mathrm{~V})$. Initial biomass concentration in two experiments was $X_{0}=0.7 \mathrm{~g} / \mathrm{L}$ and $X_{0}=1.4 \mathrm{~g} / \mathrm{L}$ respectively.

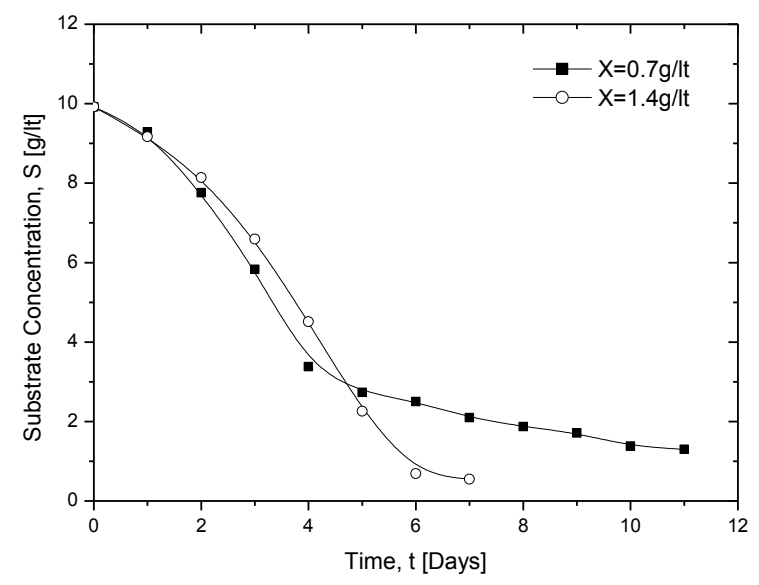

Fig. 5: Variation of substrate concentration with time when the initial biomass concentration is changed

\subsection{Performance of MEC:}

\subsubsection{Analysis of substrate response to initial biomass concentration:}

The substrate concentration was determined by titration against Hydrochloric acid as described in section 2.4. Variation of substrate concentration with time when the initial biomass concentration was varied, is shown in Fig. 5. It can be observed that the substrate consumption is increased and the time taken by the microbes to consume the substrate is decreased, due to increase in initial biomass concentration [20]. The experimental results are compared with that of simulation and the same is shown in Fig. 6 and Fig. 7 for the initial biomass concertation of $X_{0}=0.7 \mathrm{~g} / \mathrm{L}$ and $X_{0}=1.4 \mathrm{~g} / \mathrm{L}$ respectively. Results of simulation are close to that of experimental results at higher $X_{0}$. However, there is a slight deviation at lower $X_{0}$. The reason being that the equations used in the model are accurate for the exponential growth period of the microbes.

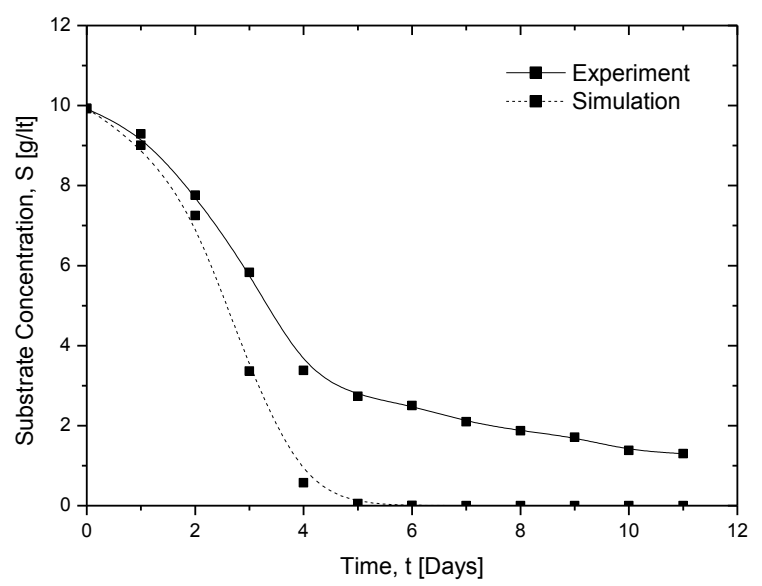

Fig. 6: Comparison of experimental results of substrate concentration variation with that of simulation at an initial biomass concertation of $X_{0}=0.7 \mathrm{~g} / \mathrm{L}$

at higher $X_{0}$, the rate of substrate consumption is faster and this result coincides with the work of [21] and most of the substrate is consumed within first 6 days of the experiment.

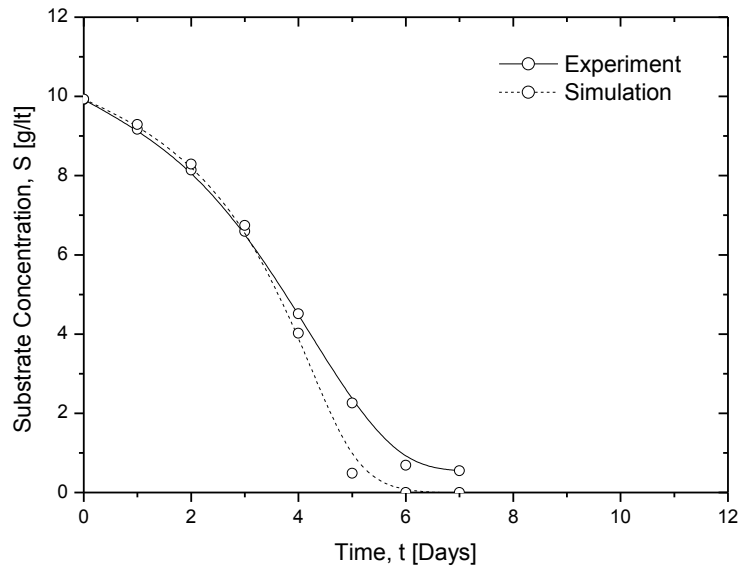

Fig. 7: Comparison of experimental results of substrate concentration variation with that of simulation at an initial biomass concertation of $X_{0}=1.4 \mathrm{~g} / \mathrm{L}$

\subsubsection{Analysis of product response to initial biomass concentration}

The quantity of hydrogen gas obtained at varying $X_{0}$ is compared and shown in Fig. 8. The observations are very similar to that of substrate consumption. At higher $X_{0}$, the product formation rate is higher and the quantity of product obtained is also slightly higher. The predictions of the model are better and closer to that of experimental results, at higher $X_{0}$. It can be construed that the Anode Respiring Bacteria grow faster at higher initial biomass concentration, and hence the rate of their metabolic activity also increases. This results in a faster 
release of protons from the biofilm leading to increase in hydrogen generation rate and conforms with the results in literature [22]. The experimental results are compared with that of simulation in Fig. 9 and Fig. 10 for $X_{0}=0.7$ $\mathrm{g} / \mathrm{L}$ and $X_{0}=1.4 \mathrm{~g} / \mathrm{L}$ respectively.

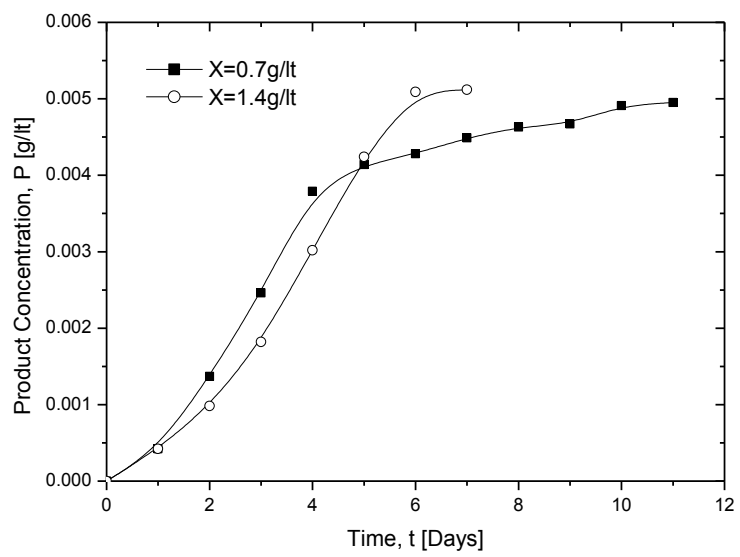

Fig. 8: Change in quantity of hydrogen gas produced with time when the initial biomass concentration is changed

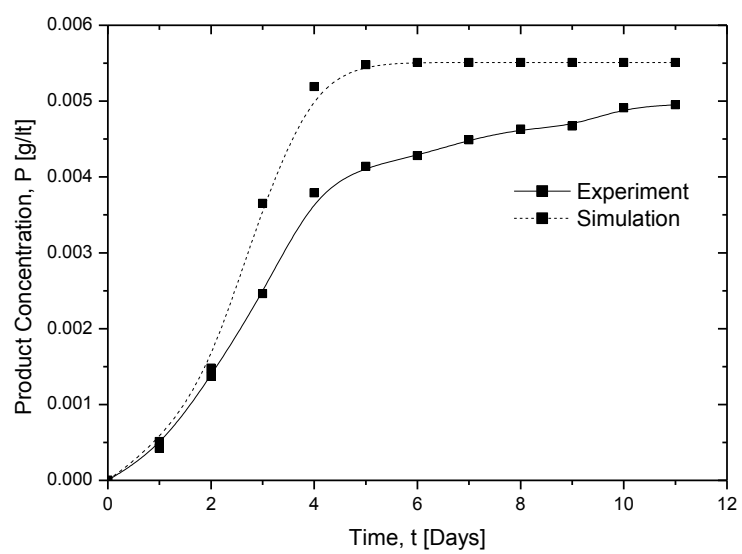

Fig. 9: Time-bound Product formation at an initial biomass concentration of $X_{0}=0.7 \mathrm{~g} / \mathrm{L}$. Experimental results are compared with that of simulation

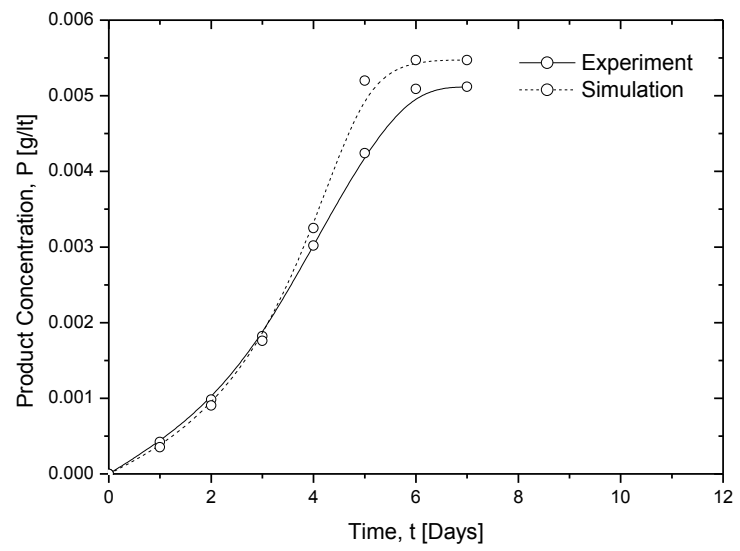

Fig. 10: Time-bound Product formation at an initial biomass concentration of $X_{0}=1.4 \mathrm{~g} / \mathrm{L}$. Experimental results are compared with that of simulation

\subsubsection{Comparison of gas volume liberated}

In terms of volume of product gases formed, the formation rate was found to be $9.42 \mathrm{ml} /$ day and 15.33 $\mathrm{ml} /$ day at initial biomass concentrations of $0.7 \mathrm{~g} / \mathrm{L} \&$ $1.4 \mathrm{~g} / \mathrm{L}$ respectively which is close to an increase of $38 \%$.

\section{Conclusion}

It can be concluded that the product formation is directly proportional to the initial biomass concentration, in a dual chamber MEC with the graphite anode and stainless-steel cathode using sodium acetate substrate and Pseudomonas aeruginosa as microbes. A cumulative gas liberation rate of $9.42 \mathrm{ml} /$ day was observed at a biomass concentration of $0.7 \mathrm{~g} / \mathrm{L}$, whereas $15.33 \mathrm{ml} /$ day was observed at a biomass concentration of $1.4 \mathrm{~g} / \mathrm{L}$. This clearly shows that an increase in the input biomass concentration leads to a significant improvement in the gas liberation rate, thereby also improving the prospects of scaling up of such a bioreactor. It was established that sodium acetate is a suitable substrate for the Pseudomonas aeruginosa microbes to grow on.

The MEC performance can be analyzed by evaluating certain parameters such as initial biomass concentration. Energy efficiency of the MEC improved substantially from $47 \%$ to $77 \%$ when the initial biomass concentration was increased from $0.7 \mathrm{~g} / \mathrm{L}$ to $1.4 \mathrm{~g} / \mathrm{L}$ respectively. The energy demand per unit volume decreased from 6.84 $\mathrm{kWh} / \mathrm{m}^{3}$ of $\mathrm{H}_{2}$ to $4.20 \mathrm{kWh} / \mathrm{m}^{3}$ of $\mathrm{H}_{2}$ due to increase in initial biomass concentration. These results are extremely encouraging from a scale-up perspective as the electricity costs can be reduced.

\section{References}

[1] WHO, WHO Cat. pub. data (2008)

[2] P. M. Doran, Bioprocess Engineering Principles (2012)

[3] M. Cai, J. Liu and Y. Wei, Env. Sci. \& Tech., 38, 11 (2004)

[4] R. A. Rozendal, H. V. M. Hamelers, G. J. W. Euverink, S. J. Metz, C. J. N. Buisman, Int. J. Hyd. Enr., 31, 12 (2006)

[5] G. Reguera, K. D. McCarthy, T. Mehta, J. Nicoli , M. Tuominen, D. Lovely, Nature, 435, (2005)

[6] B. Logan, D. Call, S. Cheng, H. Hamelers, T. H. J. A. Sleutels, R. A. Rozendal, Env. Sci. \& Tech., 42, 23 (2008)

[7] B. E. Logan, J. M. Regan, Tren. Micr. bio., 14, 12 (2006)

[8] Y. Zhao, Z. Dong, Y. Wang, J. Li, X. An, D. Yang, Int. J. Hyd. Enr., (to be published)

[9] J. Yu, M. Wu, Y. Tang, J. Shi, J. Hu, Z. Yu, J. Chen, J. Env. Sci., 86 (2019)

[10] R. Rousseau, L. Etcheverry, E. Roubaud, R. Basséguy, M.-L. Délia, A. Bergel, Appl. Enr., 257 (2019) 
[11] K. M. Hernández-García, B. Cercado, F. A. Rodríguez, F. F. Rivera, E. P. Rivero, Bio. chem. Eng. J, 162 (2020)

[12] K. Hernández-García, B. Cercado, E. Rivero, F. Rivera, Fuel, 279 (2020)

[13] H. Ren, H.-S. Lee, J. Zhang, C. L. Gardner, J. Chae, Int. J. Hyd. Enr. (to be published)

[14] K. K. Kovar, T. Egli, Micr. blgy. Mol. Blgy. Rev., 62, 3 (1998)

[15] U. S. Meda, Rakesh, L. A. R. Molayan Amritanatan, Int. J. Eng. Sci. \& Res. Tech., 4, 4 (2015)

[16] U. S. Meda, P. Kumar, A. S. Kundar, A. Kumar, H. Gowda and L. A. R. Molayan Amritanatan, As. J. Chy, 29 (2017)

[17] U. S. Meda, Rakesh, S. Ramodas, Int. J. Res. Eng. Tech., 3, 11 (2014)

[18] T. M. Leib, C. J. Pereira, J. Villadsen, Chem. Eng. Sci., 56 (2001)

[19] C. R. Melchiorsen, N. B. S. Jensen, B. Christensen, K. V. Jokumsen, J. Villadsen, Biotech. \& Bioeng., 74 (2001)

[20] S. Badwal, S. Giddey, C. Munnings, A. Bhatt, A. Hollenkamp, Front. Chy, 2, 79 (2014)

[21] B. E. Logan, S. E. Oh, S. V. Ginkel, Env. Sci. \& Tech., 36, 11 (2002)

[22] G. Kyazze, A. Popov, R. Dinsdale, S. Esteves, F. Hawkes, G. Premier, Int. J. Hyd. Enr., 35 (2010) 